\title{
Too Many versus Too Few Platelets in Patients with Hepatocellular Carcinoma: Good or Bad?
}

\author{
Teh-la Huo ${ }^{a-c}$ Po-Hong Liub, d \\ ${ }^{a}$ Department of Medical Research, Taipei Veterans General Hospital, Taipei, Taiwan; \\ ${ }^{b}$ Faculty of Medicine, National Yang-Ming University School of Medicine, Taipei, Taiwan; \\ 'Institute of Pharmacology, National Yang-Ming University School of Medicine, Taipei, \\ Taiwan; ${ }^{\mathrm{d}}$ Department of Medicine, University of Texas Southwestern Medical Center, Dallas, \\ TX, USA
}

\section{Dear Editor}

We read the paper "Association of platelet count and mean platelet volume with overall survival in patients with cirrhosis and unresectable hepatocellular carcinoma" by Dr. Scheiner et al. published in a recent issue of Liver Cancer [1] with great interest. In this elegant study including 626 patients with unresectable hepatocellular carcinoma (HCC), the authors show that thrombocytopenia was highly prevalent and associated with favorable baseline tumor characteristics: smaller tumor diameter, less extrahepatic spread and vascular invasion, and earlier Barcelona Clinic Liver Cancer stages as compared to patients with normal platelet count. Notably, thrombocytopenia was also linked with longer overall survival, and these results were confirmed in their validation cohort $(n=525)$. This study shows the potential application of platelet count as a distinct prognostic marker for HCC. Although these findings are interesting, a few issues may deserve further discussion.

We have recently investigated the role of platelet count in 3,561 HCC patients identified in Taiwan between 2002 and 2017 (unpublished data). With the definition of thrombocytopenia as platelet count $<150$ $\times 10^{9} / \mathrm{L}$ and thrombocytosis as $\geq 300 \times 10^{9} / \mathrm{L}$ at the time of diagnosis, patients with thrombocytosis more often had larger tumors and higher serum alpha-fetoprotein levels. They also had higher rates of vascular invasion ( $54 \%$ vs. $29 \%$ vs. $15 \%$ ) and metastases ( $28 \%$ vs. $12 \%$ vs. $6 \%$ ), respectively, for thrombocytosis, normal platelet, and thrombocytopenic patients. Importantly, thrombocytosis was independently associated with worse survival after adjusting for age, gender, liver disease etiology, Barcelona Clinic Liver Cancer stages, model for end-stage liver disease score, and alpha-fetoprotein level in the multivariate Cox model. However, contrary to the data by Dr. Scheiner et al., the overall survival was similar between thrombocytopenic patients and patients with normal platelet count.

It is well known that thrombocytopenia was often associated with the development of cirrhosis with or without HCC [2]. On the other hand, platelets also participate actively in almost every step of HCC carcinogenesis $[3,4]$. Consistent with this notion, antiplatelet therapy was reported to associate with a reduction in risk of liver cancer [5, 6]. Alternatively, among patients with known HCC, the use of antiplatelet therapy has been associated with lower recurrence rate and improved survival after surgery [7]. Thrombocytosis has 
Huo and Liu: Platelet in HCC

also been regarded as a para-neoplastic syndrome and was associated with poor prognosis in many types of cancer besides HCC. We do not have information if thrombocytosis was associated with a worse survival in the study by Scheiner et al., but their findings may prompt further clinical research on additive antiplatelet therapy in the management of HCC.

In summary, Scheiner et al.'s study provides new insight into the crucial role of platelets in prognostic prediction for HCC. Too many or too few platelets may well link with patient survival. Future studies from internationally collaborative cohorts are required to consolidate these issues.

\section{Statement of Ethics}

Nothing to declare.

\section{Disclosure Statement}

The authors have no conflicts of interest to declare.

\section{Funding Sources}

This study was supported by the grants from Taipei Veterans General Hospital (VN108-05 and V108C008), Taipei, Taiwan.

\section{Author Contributions}

Guarantor of the article: T.-I. Huo. Specific author contributions: T.-I. Huo and P.-H. Liu performed the research. T.-I. Huo designed the study and wrote the paper. All authors approved the final version of the manuscript.

\section{References}

1 Scheiner B, Kirstein M, Popp S, Hucke F, Bota S, Rohr-Udilova N, et al. Association of platelet count and mean platelet volume with overall survival in patients with cirrhosis and unresectable hepatocellular carcinoma. Liver Cancer. 2019 May;8(3):203-17.

2 van der Meer AJ, Feld JJ, Hofer H, Almasio PL, Calvaruso V, Fernández-Rodríguez CM, et al. Risk of cirrhosisrelated complications in patients with advanced fibrosis following hepatitis C virus eradication. J Hepatol. 2017 Mar;66(3):485-93.

3 Sitia G, Aiolfi R, Di Lucia P, Mainetti M, Fiocchi A, Mingozzi F, et al. Antiplatelet therapy prevents hepatocellular carcinoma and improves survival in a mouse model of chronic hepatitis B. Proc Natl Acad Sci USA. 2012 Aug; 109(32):E2165-72.

4 Maini MK, Schurich A. Platelets harness the immune response to drive liver cancer. Proc Natl Acad Sci USA. 2012 Aug;109(32):12840-1.

5 Lee M, Chung GE, Lee JH, Oh S, Nam JY, Chang Y, et al. Antiplatelet therapy and the risk of hepatocellular carcinoma in chronic hepatitis B patients on antiviral treatment. Hepatology. 2017 Nov;66(5):1556-69.

6 Simon TG, Ma Y, Ludvigsson JF, Chong DQ, Giovannucci EL, Fuchs CS, et al. Association between aspirin use and risk of hepatocellular carcinoma. JAMA Oncol. 2018 Dec;4(12):1683-90.

7 Lee PC, Yeh CM, Hu YW, Chen CC, Liu CJ, Su CW, et al. Antiplatelet therapy is associated with a better prognosis for patients with hepatitis B virus-related hepatocellular carcinoma after liver resection. Ann Surg Oncol. 2016 Dec;23(S5 Suppl 5):874-83. 